\title{
PLANAR MAPPINGS OF SUBEXPONENTIALLY INTEGRABLE DISTORTION: INTEGRABILITY OF DISTORTION INVERSES
}

\author{
Haiqing $\mathrm{Xu}$ \\ University of Science and Technology of China, School of Mathematical Sciences \\ Hefei 230026, P. R. China; hqxu@mail.ustc.edu.cn
}

\begin{abstract}
We establish the optimal regularity for the distortion of inverses of mappings of finite distortion with logarithm-iterated style subexponentially integrable distortion, which generalizes the Theorem 1 of [7].
\end{abstract}

\section{Introduction}

We say that a mapping $f: \Omega \rightarrow \mathbf{R}^{n}$ in a domain $\Omega \subset \mathbf{R}^{n}$ is a mapping of finite distortion, if

(i) $f \in W_{\text {loc }}^{1,1}\left(\Omega, \mathbf{R}^{n}\right)$,

(ii) the Jacobian determinant $J_{f} \in L_{\text {loc }}^{1}(\Omega)$, and

(iii) there is a measurable function $K: \Omega \rightarrow[1,+\infty]$ with $K(z)<\infty$ almost everywhere such that

$$
|D f(z)|^{n} \leq K(z) J_{f}(z) \text { for almost all } z \in \Omega
$$

where $|D f(z)|$ is the operator norm of the matrix $D f(z)$. For mappings of finite distortion, we define the distortion function by

$$
K_{f}(z)= \begin{cases}\frac{|D f(z)|^{n}}{J_{f}(z)}, & \text { if } z \in\left\{z \in \Omega: J_{f}(z)>0\right\} \\ 1, & \text { if } z \in\left\{z \in \Omega: J_{f}(z)=0\right\}\end{cases}
$$

then the distortion inequality (1.1) becomes

$$
|D f(z)|^{n}=K_{f}(z) J_{f}(z) \text { for almost every } z \in \Omega .
$$

We will limit the discussion in this paper to the planar case, i.e. $n=2$. In this case, since $|D f(z)|=\left|f_{z}\right|+\left|f_{\bar{z}}\right|$ and $J_{f}(z)=\left|f_{z}\right|^{2}-\left|f_{\bar{z}}\right|^{2}$, the distortion equality (1.2) is equivalent to the Beltrami equation

$$
\frac{\partial f(z)}{\partial \bar{z}}=\mu(z) \frac{\partial f(z)}{\partial z} \text { for almost every } z \in \Omega
$$

where $\frac{\partial}{\partial \bar{z}}=\frac{1}{2}\left(\frac{\partial}{\partial x}+i \frac{\partial}{\partial y}\right), \frac{\partial}{\partial z}=\frac{1}{2}\left(\frac{\partial}{\partial x}-i \frac{\partial}{\partial y}\right)$ and $|\mu(z)|=\frac{K_{f}(z)-1}{K_{f}(z)+1}$. For more details about mappings of finite distortion, we refer the reader to [9] and the references therein.

If $\|\mu\|_{\infty} \leq k<1$, then the classical measurable Riemann mapping theorem tells that the Beltrami equation (1.3) admits a homeomorphic solution and other solutions are represented by composing the homeomorphic solution with holomorphic functions, see $[1,3]$.

https://doi.org/10.5186/aasfm.2017.4225

2010 Mathematics Subject Classification: Primary 30C62.

Key words: Mappings of finite distortion, subexponential distortion, distortion of inverses.

This work was supported by the National Natural Science Foundation of China grants 11571333, 11471301. 
When $\|\mu\|_{\infty}=1$, the Beltrami equation (1.3) becomes degenerate. David dealt with this degenerate Beltrami equation in [4], where he generalized the measurable Riemann mapping theorem for mappings with exponentially integrable distortion $K_{f}$, i.e. for mappings with $\exp \left(p K_{f}\right) \in L_{\text {loc }}^{1}(\Omega)$ for some $p>0$. David also noted that even if the distortion of $f$ is exponentially integrable the distortion function of $f^{-1}$ might still fail to be exponentially integrable. Later, Hencl and Koskela [8] proved $K_{f^{-1}} \in L_{\text {loc }}^{\beta}$ where $\beta=c_{0} p$ with absolute constant $c_{0}$, under the local integrability of $\exp \left(p K_{f}\right)$. Based on Theorem 1.1 of [2], Gill [6] ascertained the sharp inequality $c_{0}<1$. The comprehensive statement is as follows.

Theorem A. [6, Theorem 1] Suppose that $f: \Omega \rightarrow \mathbf{C}$ is a homeomorphism of finite distortion which satisfies the Beltrami equation (1.3), with the associated distortion function $K_{f}$. If

$$
\exp \left(p K_{f}\right) \in L_{\text {loc }}^{1}(\Omega) \text { for some } p>0
$$

then $f^{-1}$ is a mapping of finite distortion and the distortion function $K_{f^{-1}}$ satisfies

$$
K_{f^{-1}} \in L_{\mathrm{loc}}^{\beta}(f(\Omega)) \text { for all } 0<\beta<p \text {. }
$$

Moreover, this result is sharp in the sense that for every $p>0$ there are functions $f$ as above such that $K_{f^{-1}} \notin L_{\text {loc }}^{p}$.

Let $p>0$, we define

$$
\mathcal{A}_{p, n}(x)= \begin{cases}p x-p, \quad \text { if } n=0, \\ \frac{p x}{1+\prod_{k=1}^{n} \log _{(k)}\left(\exp _{(k-2)}(e)-1+x\right)}-p, & \text { if } n=1,2, \ldots\end{cases}
$$

where $\log _{(i)}(x)=\log (\cdots(\log (\log (x))) \cdots)$ and $\exp _{(i)}(x)=\exp (\cdots(\exp (\exp (x))) \cdots)$ are $i$-iterated logarithm and exponent for $i=1,2, \ldots, \exp _{(0)}(x)=x$ and $\exp _{(-1)}(x)=$ 1. Theorem A tells us that the distortion function of inverse is locally integrable under $\exp \left[\mathcal{A}_{p, 0}\left(K_{f}\right)\right] \in L_{\text {loc }}^{1}$. Gill in [7] generalized Theorem 1.1 of [2] to the solution $f$ to the Beltrami equation (1.3) with $\exp \left[\mathcal{A}_{p, n}\left(K_{f}\right)\right] \in L_{\text {loc }}^{1}$ when $n=1,2, \ldots$. However, there is no corresponding result analogous to Theorem A. The aim of this article is to present a generalization of Theorem A under the local integrability of $\exp \left[\mathcal{A}_{p, n}\left(K_{f}\right)\right]$.

Theorem 1.1. Suppose that $f: \Omega \rightarrow \mathbf{C}$ is a homeomorphic mapping of finite distortion, with the associated distortion function $K_{f}$. Let $\mathcal{A}_{p, n}(x)$ be (1.4) when $n=1,2, \ldots$. If

$$
\exp \left[\mathcal{A}_{p, n}\left(K_{f}\right)\right] \in L_{\text {loc }}^{1}(\Omega) \text { for some } p>0,
$$

then $f^{-1}$ is a mapping of finite distortion and the distortion function $K_{f^{-1}}$ satisfies

$$
\log _{(n)}\left(\exp _{(n-1)}(e)+K_{f^{-1}}\right) \in L_{\text {loc }}^{\beta}(f(\Omega)) \text { for every } 0<\beta<p .
$$

Moreover, for every $p>0$ there are mappings that satisfy the assumptions of the theorem, yet fail (1.6) for $\beta=p$.

The rest of the paper is organized as follows. In section 2, we recall some basic facts about Legendre Transformation and obtain an inequality of Young type. Section 3 is devoted to the proof of Theorem 1.1.

Notation. By $s \gg 1$ and $t \ll 1$ we mean that $s$ is sufficiently large and $t$ is sufficiently small, respectively. By $f \lesssim g$ we mean that there exists a constant $M>0$ such that $f(x) \leq M g(x)$ for every $x$. If $f \lesssim g$ and $g \lesssim f$ we may denote $f \sim g$. By $\mathbf{N}$ we denote the set of positive integers. When concerned only with the convergence of improper integrals, we use notations $\int_{*}^{\infty}$ and $\int_{0}^{*}$. 


\section{An inequality of Young type}

An Orlicz function is a continuously increasing function $\Phi:[0, \infty) \rightarrow[0, \infty)$ with

$$
\Phi(0)=0 \text { and } \lim _{t \rightarrow \infty} \Phi(t)=\infty .
$$

The conjugate to an Orlicz function $\Phi$ is defined by

$$
\Phi^{*}(s)=\sup _{t \geq 0}\{s t-\Phi(t)\}, \quad s \geq 0 .
$$

Directly from definition, we obtain

$$
t s \leq \Phi(t)+\Phi^{*}(s) \text { for every } t, \quad s \geq 0 .
$$

Inspired by [5], we now impose condition

$$
\lim _{t \rightarrow \infty} \frac{\Phi(t)}{t}=\infty
$$

on the Orlicz function $\Phi$ to ensure that $\Phi^{*}(s) \in[0, \infty)$ for all $s \geq 0$. Moreover, there exists $t(s) \in[0, \infty)$ such that the supremum of $s t-\Phi(t)$ is attained at $t(s)$.

Lemma 2.1. Suppose that $\Phi$ is an Orlicz function and satisfies (2.2). If $\Phi(t)$ is differentiable for all $t \geq 0$ and $\Phi(t)$ is twice differentiable with $\Phi^{\prime \prime}(t)>0$ for all $t \gg 1$, then

$$
\left(\Phi^{*}\right)^{\prime}(s)=\left(\Phi^{\prime}\right)^{-1}(s) \text { for all } s \gg 1 .
$$

Proof. Given $s \gg 1$. From (2.2) and the continuity of $\Phi$, there exists $t(s) \in[0, \infty)$ such that

Then

$$
\Phi^{*}(s)=s t(s)-\Phi(t(s)) .
$$

$$
\Phi^{\prime}(t(s))=s .
$$

From (2.2) and $\lim _{t \rightarrow \infty} \Phi(t)=\infty$, it follows that $\lim t \rightarrow \infty \Phi^{\prime}(t)=\infty$. Hence, we have $\Phi^{\prime}(t) \gg 1$ as $t \gg 1$. Since $\Phi^{\prime \prime}(t)>0$ for all $t \gg 1$, we know that $\left(\Phi^{\prime}\right)^{-1}(s)$ exists for all $s=\Phi^{\prime}(t) \gg 1$. Applying $\left(\Phi^{\prime}\right)^{-1}$ to both sides of $(2.3)$, we have $t(s)=\left(\Phi^{\prime}\right)^{-1}(s)$. Consequently, we have

$$
\left(\Phi^{*}\right)^{\prime}(s)=t(s)+\left(s-\Phi^{\prime}(t(s))\right) \frac{d\left(\Phi^{\prime}\right)^{-1}(s)}{d s}=\left(\Phi^{\prime}\right)^{-1}(s) .
$$

The $t(s)$ in the proof of Lemma 2.1 is unique. If not, by mean value theorem there is $t_{0}(s) \gg 1$ such that $\Phi^{\prime \prime}\left(t_{0}(s)\right)=0$, which violates the assumption in Lemma 2.1.

Given a strictly convex $C^{2}$ function $\Phi(t)$, it is not easy to compute the explicit expression of $\Phi^{*}(s)$ from the definition. However, by Lemma 2.1, we can obtain the asymptotic behaviour of $\Phi^{*}(s)$ as $s \gg 1$. The following example, coming from [5], illustrates this.

Example 2.2. Put $\Phi(t)=\exp \left(\frac{t}{\log (e+t)}\right)$. Take some $t_{0} \gg 1$ such that $\Phi^{\prime}\left(t_{0}\right)>$ 0 . We define $\Phi_{1}(t)$ by

$$
\Phi_{1}(t)= \begin{cases}\Phi^{\prime}\left(t_{0}\right)\left(t-t_{0}\right)+t_{0} \Phi\left(t_{0}\right), & \text { if } 0 \leq t \leq t_{0} \\ \Phi(t)+t_{0} \Phi^{\prime}\left(t_{0}\right)-\Phi\left(t_{0}\right), & \text { if } t_{0}<t\end{cases}
$$

We first compute the asymptotic behaviour of $\Phi_{1}^{*}(s)$ as $s \gg 1$. After differentiating and taking the logarithm, we have

$$
\log \left(\Phi_{1}^{\prime}(t)\right) \sim \frac{t}{\log (t)} \text { as } t \gg 1 .
$$


Let $\frac{t}{\log (t)}=\log (s)$. Then

$$
t \sim \log (s) \log _{(2)}(s) \text { as } s \gg 1
$$

Moreover, we can check that $\Phi_{1}(t)$ satisfies all assumptions in Lemma 2.1. Hence, it follows from Lemma 2.1 that

$$
\log \left(\Phi_{1}^{\prime}(t)\right) \sim \log (s)=\log \left[\Phi_{1}^{\prime}\left(\left(\Phi_{1}^{*}\right)^{\prime}(s)\right)\right] .
$$

Therefore, by $(2.4)$ and the monotonicity of $\log (\cdot)$ and $\Phi_{1}^{\prime}(\cdot)$, we have

$$
\left(\Phi_{1}^{*}\right)^{\prime}(s) \sim \log (s) \log _{(2)}(s) .
$$

Hence, by the Newton-Leibniz formula, we show

$$
\Phi_{1}^{*}(s) \sim s \log (s) \log _{(2)}(s) \text { as } s \gg 1 .
$$

By the definition of conjugate function, we know that there exist constant $C_{1}$ and $C_{2}$ with $C_{2} \geq C_{1}$ such that $\Phi^{*}(s)+C_{1} \leq \Phi_{1}^{*}(s) \leq \Phi^{*}(s)+C_{2}$ for all $s \geq 0$. Therefore, we have

$$
\Phi^{*}(s) \sim s \log (s) \log _{(2)}(s) \text { as } s \gg 1 .
$$

By the method analogous to Example 2.2, we present an inequality of Young type, which plays a crucial role in the proof of Theorem 1.1.

Lemma 2.3. Let $p>0$ and $n \in \mathbf{N}$. Given $\beta>0$, there exist constants $C_{1}, C_{2}>$ 0 such that

$$
t s \leq C_{1} \Phi(t)+C_{2} \Psi(s) \text { for all } t, s \geq 0,
$$

where $\Phi(t)=\exp \left[\mathcal{A}_{p, n}\left(\exp _{(n)}\left(t^{\frac{1}{\beta}}\right)\right)\right]$ and $\Psi(s)=s\left[\log _{(n+1)}\left(\exp _{(n)}(e)+s\right)\right]^{\beta}$.

Proof. We divide the proof into two cases.

Case 1. Suppose first that $0 \leq s \leq C_{1}$ for some $C_{1}>0$. Since $t \leq \Phi(t)$ for all $t \geq 0$ and $\Psi(s) \geq 0$ for all $s \geq 0$, we obtain

$$
s t \leq C_{1} \Phi(t)+\Psi(s) \text { for all } t \geq 0 \text { and } 0 \leq s \leq C_{1} \text {. }
$$

Case 2. Suppose that $s \gg 1$. Take some $t_{0} \gg 1$ such that $\Phi^{\prime}\left(t_{0}\right)>0$. We define $\Phi_{1}(t)$ by

$$
\Phi_{1}(t)= \begin{cases}\Phi^{\prime}\left(t_{0}\right)\left(t-t_{0}\right)+t_{0} \Phi\left(t_{0}\right), & \text { if } 0 \leq t \leq t_{0}, \\ \Phi(t)+t_{0} \Phi^{\prime}\left(t_{0}\right)-\Phi\left(t_{0}\right), & \text { if } t_{0}<t .\end{cases}
$$

We first compute the asymptotic behaviour of $\Phi_{1}^{*}(s)$ as $s \gg 1$. Since

$$
\log \Phi_{1}^{\prime}(t) \sim \frac{\exp _{(n)}\left(t^{\frac{1}{\beta}}\right)}{\exp _{(n-1)}\left(t^{\frac{1}{\beta}}\right) \cdots \exp _{(1)}\left(t^{\frac{1}{\beta}}\right) t^{\frac{1}{\beta}}}=F(t) \text { for all } t \gg 1
$$

and for any constant $C>0$ we have

$$
C<\exp _{(n-2)}\left(t^{\frac{1}{\beta}}\right) \cdots \exp _{(1)}\left(t^{\frac{1}{\beta}}\right) t^{\frac{1}{\beta}}<C \exp _{(n-1)}\left(t^{\frac{1}{\beta}}\right) \text { as } t \gg 1
$$

it follows that

$$
\frac{\exp _{(n)}\left(t^{\frac{1}{\beta}}\right)}{\exp _{(n-1)}\left(t^{\frac{1}{\beta}}\right)}>\log \Phi_{1}^{\prime}(t)>\frac{\exp _{(n)}\left(t^{\frac{1}{\beta}}\right)}{\left[\exp _{(n-1)}\left(t^{\frac{1}{\beta}}\right)\right]^{2}} \text { for all } t \gg 1
$$


Next consider the right-hand side of (2.6). Let $b=\exp _{(n)}\left(t^{\frac{1}{\beta}}\right)$, we consider

$$
\frac{b}{[\log (b)]^{2}}=\log (s) \text {, i.e. } \frac{b^{\frac{1}{2}}}{\log \left(b^{\frac{1}{2}}\right)}=\sqrt{4 \log (s)} .
$$

By Example 2.2, we have $b^{\frac{1}{2}} \sim \sqrt{\log (s)} \log _{(2)}(s)$ as $s \gg 1$. Taking $n$ successive logarithms, we have

$$
t \sim\left[\log _{(n+1)}(s)\right]^{\beta} \text { as } s \gg 1 .
$$

Moreover, it is easy to check that $\Phi_{1}(t)$ satisfies all assumptions in Lemma 2.1. Here, we only check that $\Phi_{1}^{\prime \prime}(t)>0$ for all $t \gg 1$. By chain rule we get $F^{\prime}(t)>0$ as $t \gg 1$. Since $\lim _{t \rightarrow \infty} \frac{\log \Phi_{1}^{\prime}(t)}{F(t)}=p>0$, by L'Hospital's rule we have $\lim _{t \rightarrow \infty} \frac{\Phi_{1}^{\prime \prime}(t)}{\Phi_{1}^{\prime}(t) F^{\prime}(t)}=p>0$. Hence, we have $\Phi_{1}^{\prime \prime}(t)>0$ for all $t \gg 1$. Therefore, it follows from (2.7) and from the right-hand side of (2.6) together with Lemma 2.1 that

$$
\log \left(\Phi_{1}^{\prime}(t)\right)>\log (s)=\log \left[\Phi_{1}^{\prime}\left(\left(\Phi_{1}^{*}\right)^{\prime}(s)\right)\right] \text { for all } t \gg 1 .
$$

By (2.8) and the monotonicity of $\log (\cdot)$ and $\Phi_{1}^{\prime}(\cdot)$, we have

$$
\left(\Phi_{1}^{*}\right)^{\prime}(s) \lesssim\left[\log _{(n+1)}(s)\right]^{\beta} \text { as } s \gg 1 .
$$

We now turn to the left-hand side of (2.6). By the similar arguments used to deduce (2.9), we obtain

$$
\left[\log _{(n+1)}(s)\right]^{\beta} \lesssim\left(\Phi_{1}^{*}\right)^{\prime}(s) \text { as } s \gg 1 .
$$

Combining (2.9) and (2.10), we obtain $\left(\Phi_{1}^{*}\right)^{\prime}(s) \sim\left[\log _{(n+1)}(s)\right]^{\beta}$ as $s \gg 1$. Hence, by the Newton-Leibniz formula, we get

$$
\Phi_{1}^{*}(s) \sim s\left[\log _{(n+1)}(s)\right]^{\beta} \text { as } s \gg 1 .
$$

By the definition of conjugate function, we know that there exist constant $C_{1}$ and $C_{2}$ with $C_{2} \geq C_{1}$ such that $\Phi^{*}(s)+C_{1} \leq \Phi_{1}^{*}(s) \leq \Phi^{*}(s)+C_{2}$ for all $s \geq 0$. Therefore, we have

$$
\Phi^{*}(s) \sim s\left[\log _{(n+1)}(s)\right]^{\beta}<\Psi(s) \text { as } s \gg 1 .
$$

It follows from (2.1) and (2.11) that there exists constant $C_{2}>0$ such that

$$
t s \leq \Phi(t)+C_{2} \Psi(s) \text { for all } t \geq 0 \text { and all } s \gg 1 .
$$

Combining (2.5) and (2.12), we complete the proof.

\section{Proof of Theorem 1.1}

We begin with four lemmas.

Lemma 3.1. [11, Theorem 1.1] Suppose that $\Psi$ is a strictly increasing, differentiable function and satisfies

$$
\begin{gathered}
\int_{1}^{\infty} \frac{\Psi^{\prime}(t)}{t} d t=\infty \\
\lim _{t \rightarrow \infty} t \Psi^{\prime}(t)=\infty
\end{gathered}
$$

Let $f: \Omega \rightarrow \mathbf{R}^{n}$ be a mapping of finite distortion and the distortion function $K_{f}$ satisfies $\exp \left(\Psi\left(K_{f}\right)\right) \in L_{\text {loc }}^{1}(\Omega)$. Then $f$ satisfies the Lusin's condition $(N)$, i.e. $f(E)$ has Lebesgue measure zero if $E$ has Lebesgue measure zero. 
Given a mapping $f: \Omega \rightarrow \mathbf{R}^{n}$, we denote $N(f, \Omega, y)$ by the number of preimages of point $y$ in $\Omega$ under $f$. We say $f$ has essentially bounded multiplicity, if $N(f, \Omega, y)$ is bounded for a.e. $y \in \mathbf{R}^{n}$.

From the proof of Theorem 1.2 in [12], we know the assertion of Theorem 1.2 in [12] remains valid if both the mapping and its distortion function lie in local Sobolev spaces. So, we have the following result:

Lemma 3.2. Let $f: \Omega \rightarrow \mathbf{R}^{2}$ be a mapping of finite distortion and the distortion function $K_{f}$ satisfies $K_{f} \in L_{\mathrm{loc}}^{1}(\Omega)$. If $f$ has essentially bounded multiplicity and $f$ is not a constant, then $J_{f}>0$ almost everywhere in $\Omega$.

Suppose that a function $\mathcal{A}$ has the properties:

$(\mathrm{A}-1) \quad \mathcal{A}:[1, \infty) \rightarrow[0, \infty)$ is a smooth increasing function with $\mathcal{A}(1)=0$.

$$
\int_{1}^{\infty} \frac{\mathcal{A}(t)}{t^{2}} \mathrm{~d} t=\infty
$$

The associated function of $\mathcal{A}$ is denoted by

$$
P(t)= \begin{cases}t^{2}, & 0 \leq t \leq 1, \\ \frac{t^{2}}{\mathcal{A}^{-1}\left(\log t^{2}\right)}, & t \geq 1 .\end{cases}
$$

Let us recall the notation

$$
W_{\text {loc }}^{1, P}(\Omega)=\left\{f \in W_{\text {loc }}^{1,1}(\Omega): P(|D f|) \in L_{\text {loc }}^{1}(\Omega)\right\} .
$$

Lemma 3.3. [3, Theorem 20.5.1] Given a function $\mathcal{A}$ satisfying $(A-1)$ and (A-2) and the associated function $P$ which is defined by (3.1). Let $f: \Omega \rightarrow \mathbf{R}^{2}$ be a mapping of finite distortion such that the distortion function $K_{f}$ satisfies $\exp \left[\mathcal{A}\left(K_{f}\right)\right] \in L_{\text {loc }}^{1}(\Omega)$, then

$$
f \in W_{\text {loc }}^{1, P}(\Omega) .
$$

Obviously, $\mathcal{A}_{p, n}$ satisfies $(\mathrm{A}-1)$ and $(\mathrm{A}-2)$. We denote the associated function of $\mathcal{A}_{p, n}$ by $P_{n}$. Next we present a lemma essentially due to Gill [7].

Lemma 3.4. Let $p>0$ and $n \in \mathbf{N}$. Given a Beltrami equation (1.3) with compactly supported $\mu(z)$, and $|\mu(z)|<1$ almost everywhere with $\exp \left[\mathcal{A}_{p, n}\left(\frac{1+|\mu(z)|}{1-|\mu(z)|}\right)\right] \in$ $L_{\text {loc }}^{1}(\mathbf{C})$. Then any solution $f \in W_{\text {loc }}^{1, P_{n}}(\Omega)$ to this Beltrami equation in a domain $\Omega \subset \mathbf{C}$ admits

$$
J_{f}\left[\log _{(n+1)}\left(\exp _{(n)}(e)+J_{f}\right)\right]^{\beta} \in L_{\mathrm{loc}}^{1}(\Omega) \text { for all } 0<\beta<p .
$$

Proof of Theorem 1.1. Since

$$
\frac{1}{\log _{(1)}(x) \log _{(2)}(x) \cdots \log _{(n)}(x)} \lesssim \mathcal{A}_{p, n}^{\prime}(x) \text { as } x \gg 1,
$$

we know $\mathcal{A}_{p, n}(x)$ satisfies $(\mathrm{C}-1)$ and $(\mathrm{C}-2)$. It follows from Lemma 3.1 that $f$ satisfies the Lusin's condition $(\mathrm{N})$.

Since

$$
x \lesssim \exp \left(\mathcal{A}_{p, n}(x)\right) \text { for all } x \geq 1,
$$

it follows from (1.5) that

$$
K_{f} \in L_{\mathrm{loc}}^{1}(\Omega) .
$$

So, Lemma 3.2 tells us $J_{f}>0$ almost everywhere in $\Omega$. 
Given compact set $\widetilde{M} \subset f(\Omega)$, we have $M=f^{-1}(\widetilde{M}) \subset \Omega$ is a compact set. By Corollary 3.3.3 in [3], we obtain that $f$ is differentiable almost everywhere in $\Omega$. So, we can divide the set $M$ into two subsets $M^{\prime}$ and $M^{\prime \prime}$, where $M^{\prime}$ is the subset in which $f$ is differentiable and $J_{f}(z)>0$ and $M^{\prime \prime}=M \backslash M^{\prime}$ has Lebesgue measure zero. For any $z \in M^{\prime}$, by Lemma A.29 of [9], we have

$$
D f^{-1}(f(z))=(D f(z))^{-1} .
$$

Hence, by Cramer's rule we have $\left|D f^{-1}(f(z))\right|^{2} J_{f}(z)=K_{f}(z)$ and $K_{f^{-1}}(f(z))=$ $K_{f}(z)$ for all $z \in M^{\prime}$. So, it follows from Corollary A.36 (c) of [9] and the Lusin's condition $(\mathrm{N})$ of $f$ that

$$
\int_{\widetilde{M}}\left|D f^{-1}(w)\right|^{2} \mathrm{~d} w=\int_{M} K_{f}(z) \mathrm{d} z
$$

and

$$
\int_{\widetilde{M}}\left[\log _{(n)}\left(\exp _{(n-1)}(e)+K_{f^{-1}}\right)\right]^{\beta} \mathrm{d} w=\int_{M}\left[\log _{(n)}\left(\exp _{(n-1)}(e)+K_{f}\right)\right]^{\beta} J_{f} \mathrm{~d} z
$$

By (3.2) and $J_{f^{-1}} \leq\left|D f^{-1}\right|^{2}$, it follows from (3.3) that $J_{f^{-1}} \in L_{\text {loc }}^{1}(f(\Omega))$. Therefore, by [8, Theorem 3.3] $f^{-1}$ is a mapping of finite distortion.

Next we prove (1.6). Because of (3.4), it suffices to prove

$$
\int_{M}\left[\log _{(n)}\left(\exp _{(n-1)}(e)+K_{f}(z)\right)\right]^{\beta} J_{f}(z) \mathrm{d} z<\infty
$$

for any compact set $M \subset \Omega$. Let

$$
s=J_{f}(z) \text { and } t=\left[\log _{(n)}\left(\exp _{(n-1)}(e)+K_{f}(z)\right)\right]^{\beta} .
$$

Since

$$
\mathcal{A}_{p, n}\left(\exp _{(n)}\left(t^{\frac{1}{\beta}}\right)\right) \leq \mathcal{A}_{p, n}\left(K_{f}(z)\right)+p\left(\exp _{(n-1)}(e)-1\right),
$$

it follows from Lemma 2.3 that there exist constants $C^{\prime}$ and $C^{\prime \prime}$ such that

$$
t s \leq C^{\prime} \exp \left[\mathcal{A}_{p, n}\left(K_{f}\right)\right]+C^{\prime \prime} J_{f}\left[\log _{(n+1)}\left(\exp _{(n)}(e)+J_{f}\right)\right]^{\beta} .
$$

Note that $\mathcal{A}_{p, n}(x)$ satisfies $(\mathrm{A}-1)$ and $(\mathrm{A}-2)$ conditions, and thus Lemma 3.3 implies

$$
f \in W_{\mathrm{loc}}^{1, P_{n}}(\Omega),
$$

where $P_{n}$ is the associated function of $\mathcal{A}_{p, n}$. So, it follows from Lemma 3.4 that

$$
J_{f}\left[\log _{(n+1)}\left(\exp _{(n)}(e)+J_{f}\right)\right]^{\beta} \in L_{\mathrm{loc}}^{1}(\Omega) .
$$

Hence, according to (3.6), (1.5) and (3.7), (3.5) is proved.

To show Theorem 1.1 is sharp, as in Theorem 4 of [7], we consider Kovalev-type function $h$ in $\Omega=\mathbf{D}$ as

$$
h(z)=\frac{z}{|z|} \rho(|z|)
$$

where $\rho(t)=\left[\log _{(n+1)}\left(\exp _{(n+1)}(e)+\frac{1}{t}\right)\right]^{-\frac{p}{2}}\left[\log _{(n+2)}\left(\exp _{(n+1)}(e)+\frac{1}{t}\right)\right]^{-\frac{1}{2}}, p>0$ and $n \in \mathbf{N}$. For the reader's convenience, we carry out the main computation. By (3.4), it is enough to check

$$
J_{h}\left[\log _{(n)}\left(\exp _{(n-1)}(e)+K_{h}\right)\right]^{p} \notin L_{\mathrm{loc}}^{1}(\mathbf{D}) .
$$


From the definition of $h$, it is sufficient to consider $h$ in the small enough neighbourhood of 0. So with the formulas in section 6.5 .1 of [10], when $|z| \ll 1$, we have

$$
J_{h}(z) \sim \frac{1}{|z|^{2}} \frac{1}{\log _{(1)}\left(\frac{1}{|z|}\right)} \cdots \frac{1}{\log _{(n)}\left(\frac{1}{|z|}\right)}\left[\log _{(n+1)}\left(\frac{1}{|z|}\right)\right]^{-p-1}\left[\log _{(n+2)}\left(\frac{1}{|z|}\right)\right]^{-1}
$$

and

Since

$$
K_{h}(z)=\frac{\rho(|z|)}{|z| \rho^{\prime}(|z|)} \sim \log _{(1)}\left(\frac{1}{|z|}\right) \log _{(2)}\left(\frac{1}{|z|}\right) \cdots \log _{(n+1)}\left(\frac{1}{|z|}\right)
$$

$$
\log \left(\exp _{(n-1)}(e)+K_{h}(z)\right) \sim \log \left(K_{h}(z)\right) \sim \log _{(2)}\left(\frac{1}{|z|}\right) \quad \text { as }|z| \ll 1,
$$

we get

$$
\left[\log _{(n)}\left(\exp _{(n-1)}(e)+K_{h}\right)\right]^{p} \sim\left[\log _{(n+1)}\left(\frac{1}{|z|}\right)\right]^{p} \quad \text { as }|z| \ll 1 .
$$

Combining (3.10) and (3.11), we obtain

$$
J_{h}\left[\log _{(n)}\left(\exp _{(n-1)}(e)+K_{h}\right)\right]^{p} \sim \frac{1}{|z|^{2}} \frac{1}{\log _{(1)}\left(\frac{1}{|z|}\right)} \cdots \frac{1}{\log _{(n+2)}\left(\frac{1}{|z|}\right)}
$$

Now, (3.9) is obtained from

$$
\begin{aligned}
\int_{0}^{*} \frac{1}{t} \frac{1}{\log _{(1)}\left(\frac{1}{t}\right)} \cdots \frac{1}{\log _{(n+2)}\left(\frac{1}{t}\right)} \mathrm{d} t & =\int_{*}^{+\infty} \frac{1}{s} \frac{1}{\log _{(1)}(s)} \cdots \frac{1}{\log _{(n+2)}(s)} \mathrm{d} s \\
& =\cdots=\int_{*}^{+\infty} \frac{1}{\log (x)} \mathrm{d} x=\infty .
\end{aligned}
$$

The proof is complete.

Acknowledgements. The author wishes to express his sincere appreciation to his supervisor Professor Congwen Liu who critically read the manuscript and made numerous helpful suggestions, and to Professor Luigi Greco who provided the valuable method for Example 2.2. The author is greatly indebted to Professor James T. Gill for many useful comments. The author would like to thank the referee for careful reading, and a great variety of helpful comments and suggestions, from which he has learnt a lot.

\section{References}

[1] Ahlfors, L. V.: Lectures on quasiconformal mappings. Second edition. - Univ. Lecture Ser. 38, Amer. Math. Soc., Providence, RI, 2006.

[2] Astala, K., J. T. Gill, S. Rohde, and E. Saksman: Optimal regularity for planar mappings of finite distortion. - Ann. Inst. H. Poincaré Anal. Non Linéaire 27:1, 2010, 1-19.

[3] Astala, K., T. Iwaniec, and G. Martin: Elliptic partial differential equations and quasiconformal mappings in the plane. - Princeton Math. Ser. 48, 2009.

[4] David, G.: Solutions de l'équation de Beltrami avec $\|\mu\|_{\infty}=1$. - Ann. Acad. Sci. Fenn. Ser. A I Math. 13:1, 1988, 25-70.

[5] Giannetti, F., L. Greco, and A. Passarelli di Napoli: The self-improving property of the Jacobian determinant in Orlicz spaces. - Indiana Univ. Math. J. 59:1, 2010, 91-114.

[6] GiLL, J. T.: Integrability of derivatives of inverses of maps of exponentially integrable distortion in the plane. - J. Math. Anal. Appl. 352:2, 2009, 762-766. 
[7] Gill, J. T.: Planar maps of sub-exponential distortion. - Ann. Acad. Sci. Fenn. Math. 35:1, 2010, 197-207.

[8] Hencl, S., and P. Koskela: Regularity of the inverse of a planar Sobolev homeomorphism. - Arch. Ration. Mech. Anal. 180:1, 2006, 75-95.

[9] Hencl, S., and P. Koskela: Lectures on mappings of finite distortion. - Lecture Notes in Math. 2096, Springer, 2014.

[10] Iwaniec, T., and G. Martin: Geometric function theory and non-linear analysis. - Oxford Univ. Press, 2001.

[11] Kauhanen, J., P. Koskela, J. Malý, J. Onninen, and X. Zhong: Mappings of finite distortion: sharp Orlicz-conditions. - Rev. Mat. Iberoam. 19:3, 2003, 857-872.

[12] Koskela, P., and J. MalÝ: Mappings of finite distortion: the zero set of the Jacobian. - J. Eur. Math. Soc. 5:2, 2003, 95-105.

Received 8 April 2016 • Accepted 2 September 2016 